\title{
Measurements of inclusive WW and WZ production with ATLAS
}

\author{
Valerie S. Lang* \\ On behalf of the ATLAS Collaboration \\ Deutsches Elektronen-Synchrotron, Notkestr. 85, D-22607 Hamburg, Germany \\ E-mail: valerie.langecern.ch
}

\begin{abstract}
Measurements of electroweak boson pair production at the LHC constitute a stringent test of the electroweak sector and provide a model-independent means to search for new physics at the $\mathrm{TeV}$ scale. Recent results from ATLAS for inclusive WW and WZ production in proton-proton collisions at $\sqrt{s}=13 \mathrm{TeV}$ are presented, including polarisation studies in the WZ final state. The precision measurements are compared to theoretical predictions at NLO and NNLO in perturbative QCD.
\end{abstract}

XXVII International Workshop on Deep-Inelastic Scattering and Related Subjects - DIS2019 8-12 April, 2019

Torino, Italy

${ }^{*}$ Speaker. 


\section{Introduction}

The electroweak sector in the Standard Model (SM) of particle physics is a unique window to increase our understanding of the early universe and to search for physics beyond the SM (BSM) at the TeV scale. It describes the interactions of $W$ and $Z$ bosons by coupling to the weak isospin of particles - not only other particles, but also themselves - as a result of the weak isospin of $W$ bosons themselves. The non-abelian nature of electroweak interactions allows for triple (TGC) and quartic gauge couplings (QGC) in combinations as $W W Z, W W Z Z$ and $W W W W$. The vertex $W W \gamma$ is also possible as a result of the existence of the $W$ boson electromagnetic charge to which the photon can couple. A production mode including the triple vertex $W W Z$ contributes to both $W W$ and $W Z$ production at the Large Hadron Collider (LHC), making these processes especially interesting to probe electroweak interactions and to look for anomalous contributions to this vertex.

The electroweak sector is moreover uniquely tied to the Higgs sector in the SM by the masses of $W$ and $Z$ bosons. The weak gauge bosons obtain their masses through coupling to the Higgs field, and the finite masses of $W$ and $Z$ bosons allow for a third polarisation state - the longitudinal one - in addition to the two transverse polarisations of massless spin-1 particles. Depending on the polarisation, the bosons are produced preferrably in specific kinematic phase space regions, which means that the contributions from each polarisation state can be extracted from the measurement of dedicated differential distributions, such as in $W Z$ events.

Insights into the electroweak sector from recent measurements of $W W$ [1] and $W Z$ [2] production with the ATLAS [3] detector are discussed in the following.

\section{Measurement of WW production in ATLAS}

The production of pairs of oppositely charged $W$ bosons at the LHC is measured in $p p$ collisions at a centre-of-mass energy of $\sqrt{s}=13 \mathrm{TeV}$, recorded in 2015 and 2016 and corresponding to an integrated luminosity of $36.1 \mathrm{fb}^{-1}$ [1]. The $W$ bosons are required to decay leptonically as $W W \rightarrow e v \mu v$; the final state therefore contains one oppositely charged electron-muon pair and missing transverse energy $\left(E_{\mathrm{T}}^{\mathrm{miss}}\right)$.

The $W W$ signal is produced through $q q$ and $g g$ initial states. In the first case, production occurs both through $t$ - and $s$-channel production where in the $s$-channel the intermediate $Z / \gamma^{*}$ couples to the two $W$ bosons via the $W W Z / \gamma^{*}$ vertex. The $g g$ initial state leads to $W W$ production via a nonresonant quark loop or through a top-quark loop followed by an intermediate Higgs boson. Both $g g$ production modes can interfere, reducing the cross section - an effect that is particular relevant in high-energy tails of kinematic distributions. The $g g$ initial state contributes only about $5 \%$ to the total production cross section, with the resonant production being kinematically suppressed by selection requirements.

Events are further selected by requiring that there be no jet of particles, reconstructed with the anti- $k_{t}$ algorithm with radius parameter $R=0.4$, and with a transverse momentum $\left(p_{\mathrm{T}}\right)$ above $35 \mathrm{GeV}$ and absolute pseudorapidity $(|\eta|)$ below 4.5 . Furthermore, there must not be any jets with $p_{\mathrm{T}}>20 \mathrm{GeV}$ and $|\eta|<2.5$ that are tagged to contain $b$-quark mesons or baryons. Both jetrelated requirements are needed to suppress $W W$ events which originate from pair production of top-quarks $(t \bar{t})$ or from single-top production associated with a single $W$ boson $(W t)$. These two 
production modes are considered as background and are subtracted prior to the determination of the fiducial cross section. The first jet-related requirement is particularly restrictive in the selection of events in data, such that it is also kept as requirement in the fiducial phase space.

With the selection criteria applied, 12659 events in data pass the $W W$ selection. The obtained signal purity is about $64 \%$, with the largest background contribution of about $26 \%$ coming from $t \bar{t}+W t$ events. Further backgrounds include $W+$ jets, $Z \rightarrow \tau \tau \rightarrow e \mu+4 v$ and other di- and triboson production processes.

The fiducial cross section is determined as:

$$
\sigma_{W W}^{\mathrm{fid}}=\frac{N_{\mathrm{data}}-N_{\mathrm{bkg}}}{C_{W W} \cdot \mathscr{L}_{\mathrm{int}}}
$$

where $N_{\text {data (bkg) }}$ is the number of events selected in data (estimated as background), $C_{W W}$ corrects for detector acceptance, inefficiencies, resolution effects and $\tau$-lepton decays and is determined from $W W$ simulation. $\mathscr{L}_{\text {int }}$ is the integrated luminosity of $36.1 \mathrm{fb}^{-1}$ recorded in 2015 and 2016, measured with an uncertainty of $2.1 \%$. Differential cross sections are obtained using iterative Bayesian unfolding $[9,10]$.

The measured fiducial $W W$ production cross section is shown in Figure 1 in comparison to three variants of the next-to-next-to-leading order (NNLO) prediction from the MATRIX code [48]. The total uncertainty in the measurement is $7.1 \%$ - the most precise measurement of the $W W$ fiducial cross section at the LHC so far. The three MATRIX variants are all NNLO in the $q q$ initial state, and are complemented with a leading order (LO) prediction for the $g g$ initial state contribution, a next-to-leading order (NLO) prediction for $g g$ or the same, but including also NLO electroweak radiation (NLO EW) effects. In all three cases, the predictions are slightly below the data, but still within measurement uncertainties. While the NLO $g g$ calculation increases the cross section, the NLO EW corrections reduce the prediction, leading to the largest difference to the measured cross section among the three predictions.

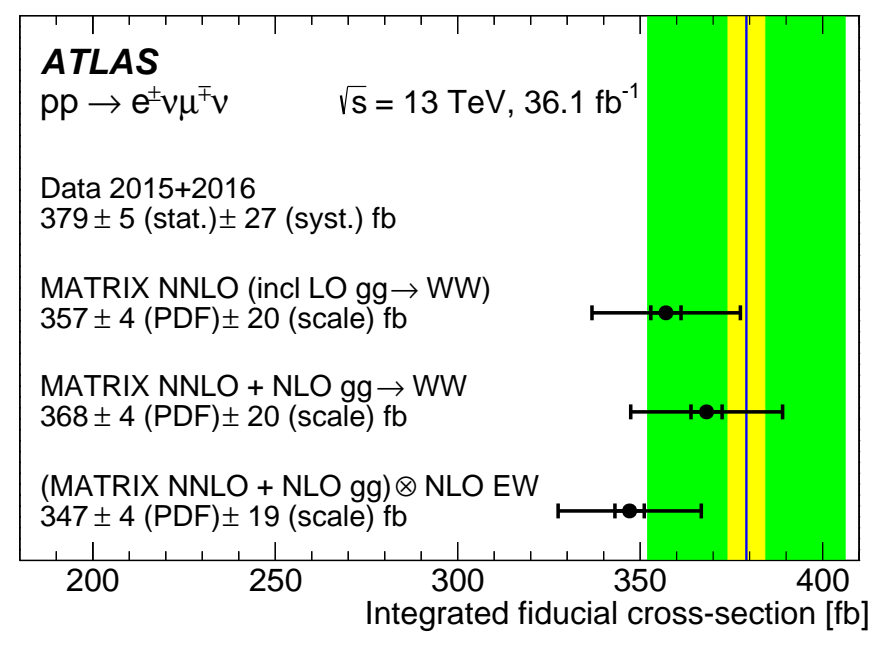

Figure 1: Measured fiducial cross section with statistical (yellow band) and systematic (green band) uncertainties, in comparison to three variants of the NNLO prediction from the MATRIX calculation [1]. 
Six differential distributions related to the $W W$ system are measured; the cross section as a function of the leading lepton transverse momentum $\left(p_{\mathrm{T}}^{\text {lead } \ell}\right)$ is displayed in Figure 2. Among the measured distributions, $p_{\mathrm{T}}^{\text {lead } \ell}$ is the most sensitive to electroweak gauge structure and the triple gauge coupling, since the $W$ polarisation in $W W$ events determines the lepton decay angle in the $W$ rest frame and hence the lepton $p_{\mathrm{T}}$ in the laboratory frame. The measurement uncertainties range from about $7 \%(5 \%)$ at low $p_{\mathrm{T}}^{\text {lead } \ell}$ to about $26 \%(25 \%)$ at the highest $p_{\mathrm{T}}^{\text {lead } \ell}$ for the absolute (normalized) cross section. The measurement uncertainty is dominated in particular at the highest $p_{\mathrm{T}}^{\text {lead } \ell}$ by the statistical uncertainty in the data. The measurement is compared to the NNLO MATRIX calculation, including the NLO $g g$ contribution and NLO EW effects, as well as three NLO $q q$ generators, interfaced to a parton shower simulation, and combined with a LO $g g$ simulation with parton shower. The predictions agree mostly within uncertainties with the data, though some shape differences are visible in particular between $100 \mathrm{GeV}$ to $300 \mathrm{GeV}$ in $p_{\mathrm{T}}^{\text {lead } \ell}$.
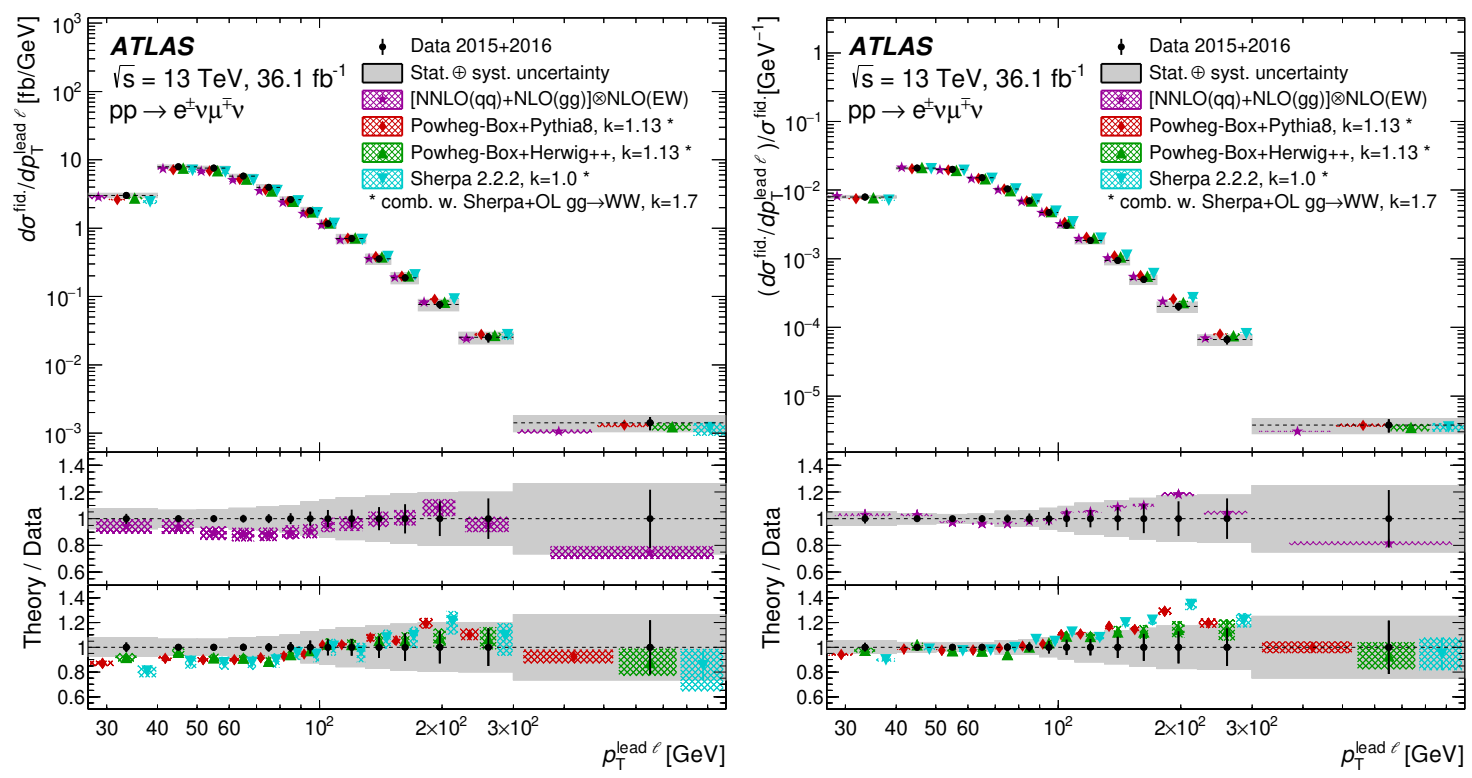

Figure 2: Fiducial $W W$ production cross section as a function of the leading lepton $p_{\mathrm{T}}$ as absolute cross section (left) and normalized to the integrated fiducial cross section (right). In both cases, the measurement is compared to the NNLO MATRIX calculation, including the NLO $g g$ contribution and NLO EW effects, as well as three NLO $q q$ generators, interfaced to a parton shower simulation, and combined with a LO $g g$ simulation with parton shower [1].

\section{Measurement of WZ production in ATLAS}

The production of $W Z$ boson pairs is measured based on the same dataset as used for the $W W$ measurement, recorded in 2015 and 2016 [2]. The $W Z$ decays considered in this measurement are the lepton combinations $e v e e, e v \mu \mu, \mu v e e$ and $\mu \nu \mu \mu$, where reconstructed leptons are assigned to originate from the $W$ or $Z$ boson according to proximity of the invariant mass of the same-flavor opposite-sign lepton pair to the $Z$ boson mass. In contrast to the $W W$ measurement, the presence of jets with $p_{T}>25 \mathrm{GeV}$ and $|\eta|<2.5$ is allowed. 

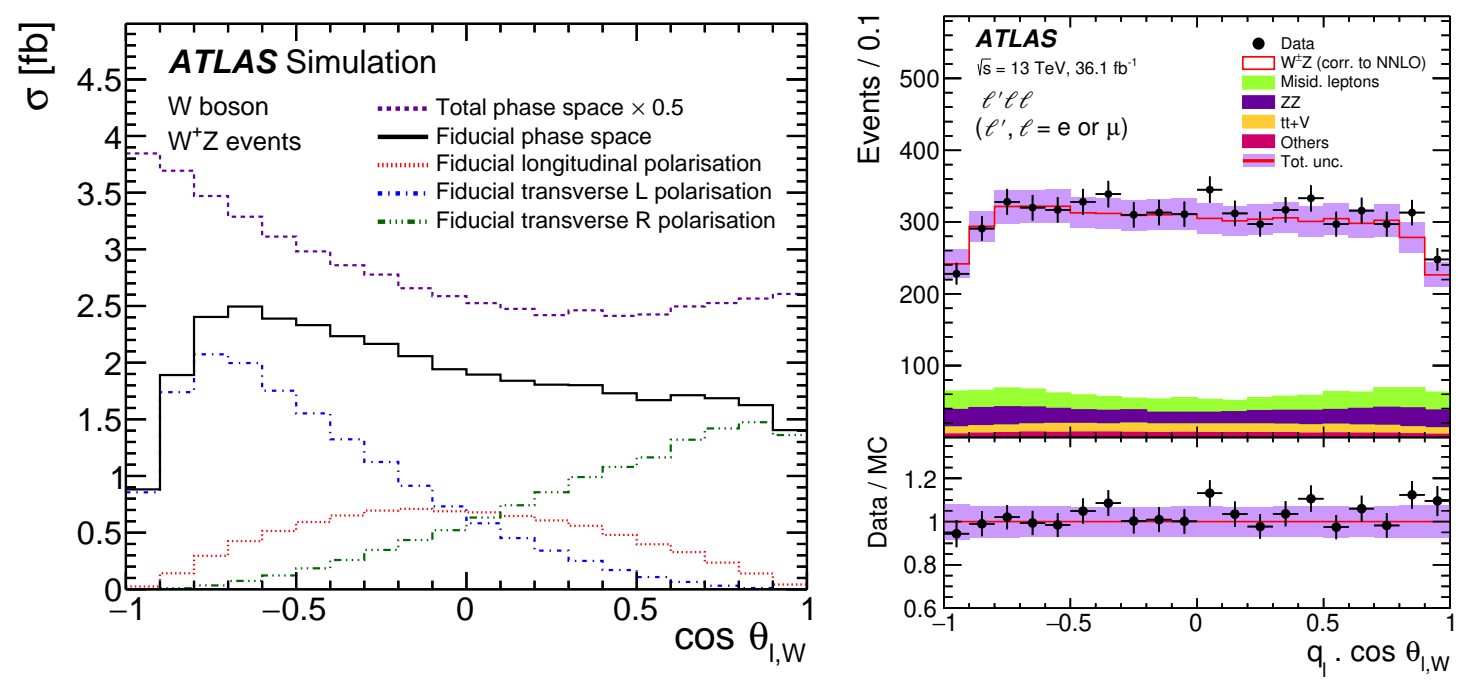

Figure 3: Total and fiducial simulated production cross section for $W^{+} Z$ events as a function of the angular variable $\cos \theta_{\ell, W}$, detailing the contributions from different $W$ polarisations, (left) and the measured $q_{\ell}$. $\cos \theta_{\ell, W}$ distribution in data, including background estimates (right). The latter is used to fit the different polarisation distributions [2].

In total, 6160 events are selected in the data, $78 \%$ of which are predicted to arise from $W Z$ production. The fiducial cross section for $W^{ \pm} Z$ production is measured with a precision of $4.5 \%$ and the ratio of $W^{+} Z$ to $W^{-} Z$ production is found to be $1.47 \pm 0.05$ for all four lepton decay channels combined. Six differential distributions are measured related to the $W Z$ system and two further distributions are determined considering the jet recoil, including the jet multiplicity.

A unique result at a hadron collider is the measurement of the longitudinal polarisation of the $W$ and $Z$ bosons in $W Z$ events. The simulated cross section for each polarisation state varies according to the angle $\theta_{\ell, W(Z)}$ between the (negatively charged) lepton in the rest frame of the $W$ $(Z)$ boson and the direction of the $W(Z)$ boson in the $W Z$ rest frame, as shown in Figure 3 (left) for the $W$ boson in $W^{+} Z$ events. The distributions $q_{\ell} \cdot \cos \theta_{\ell, W}$ and $\cos \theta_{\ell, Z}$ are measured in data, as shown in Figure 3 (right) for $q_{\ell} \cdot \cos \theta_{\ell, W}$, combining all four decay channels of the $W Z$ events. The longitudinal polarisation fraction $f_{0}$, the difference between left- and right-handed helicity transverse polarisations $f_{\mathrm{L}}-f_{\mathrm{R}}$ and the integrated fiducial cross section are then extracted in a binned profile likelihood fit to these two distributions, using templates for the three helicity states.

The extracted values are corrected for detector inefficiencies and QED final-state radiation. The obtained $W$ and $Z$ polarisation fractions are shown in Figure 4. Evidence for longitudinallypolarised $W$ bosons in $W Z$ events is obtained with $4.2 \sigma$ significance ( $3.8 \sigma$ expected) and longitudinally-polarised $Z$ bosons are observed with $6.5 \sigma$ significance $(6.1 \sigma$ expected), in agreement with the SM prediction.

\section{Conclusions}

Production of $W W$ and $W Z$ boson pairs has been measured by ATLAS in $p p$ collisions at a centre-of-mass energy of $\sqrt{s}=13 \mathrm{TeV}$ at the LHC, using data corresponding to an integrated 

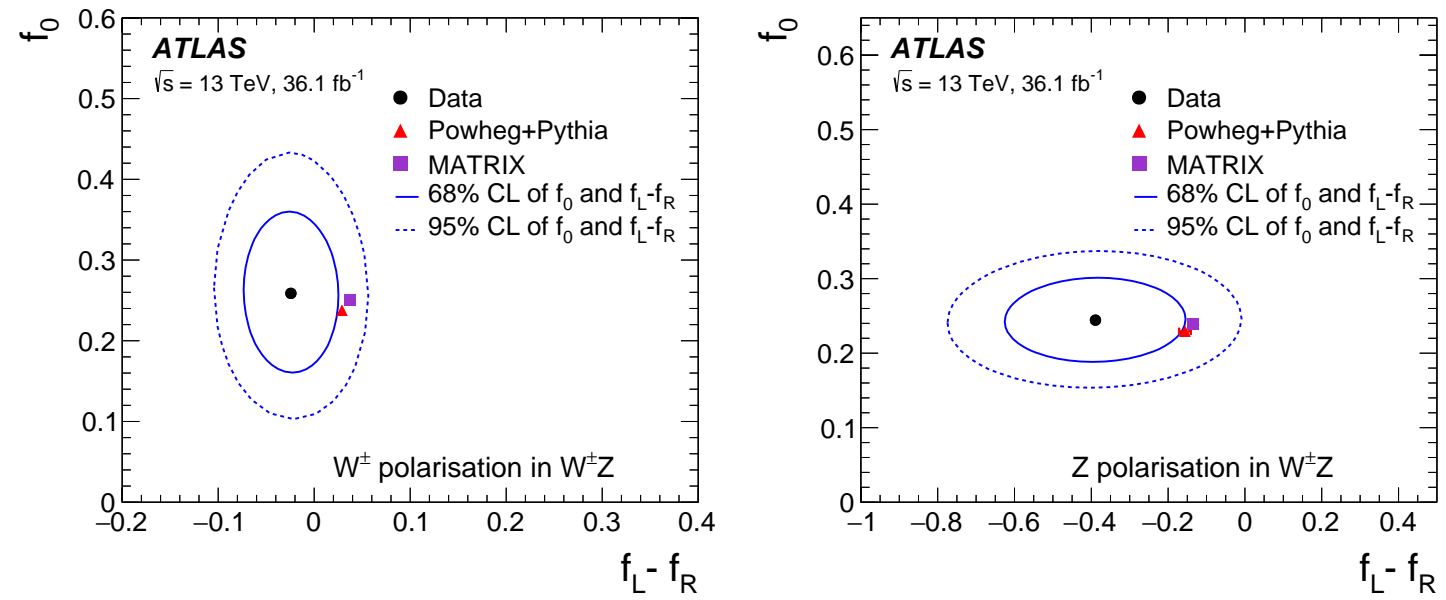

Figure 4: Measured helicity fractions $f_{0}$ vs. $f_{\mathrm{L}}-f_{\mathrm{R}}$ for $W$ (left) and $Z$ (right) bosons in $W^{ \pm} Z$ events [2].

luminosity of $36.1 \mathrm{fb}^{-1}$. The measurements probe the electroweak sector of the Standard Model, testing up-to-date predictions both for integrated and differential fiducial cross sections as well as determining for the first time in hadronic collisions the longitudinal polarisations of $W$ and $Z$ bosons in $W Z$ events. More data, recorded in 2017 and 2018, will advance these measurements further.

\section{References}

[1] ATLAS Collaboration, Measurement of fiducial and differential $W^{+} W^{-}$production cross-sections at $\sqrt{s}=13 \mathrm{TeV}$ with the ATLAS detector, 2019, hep-ex/1905.04242

[2] ATLAS Collaboration, Measurement of $W^{ \pm} Z$ production cross sections and gauge boson polarisation in pp collisions at $\sqrt{s}=13 \mathrm{TeV}$ with the ATLAS detector, 2019, hep-ex/1902.05759

[3] ATLAS Collaboration, The ATLAS Experiment at the CERN Large Hadron Collider, JINST 3 (2008) S08003

[4] M. Grazzini, S. Kallweit and M. Wiesemann, Fully differential NNLO computations with MATRIX, Eur. Phys. J. C 78 (2018) 537 [hep-ph/1711.06631]

[5] F. Cascioli, P. Maierhofer and S. Pozzorini, Scattering Amplitudes with Open Loops, Phys. Rev. Lett. 108 (2012) 111601 [hep-ph/1111.5206]

[6] T. Gehrmann, A. von Manteuffel and L. Tancredi, The two-loop helicity amplitudes for $q \bar{q}^{\prime} \rightarrow V_{1} V_{2} \rightarrow 4$ leptons, JHEP 09 (2015) 128 [hep-ph/1503.04812]

[7] F. Caola, M. Dowling, K. Melnikov, R. Röntsch and L. Tancredi, QCD corrections to vector boson pair production in gluon fusion including interference effects with off-shell Higgs at the LHC, JHEP 07 (2016) 087 [hep-ph/1605.04610]

[8] B. Biedermann et al., Next-to-leading-order electroweak corrections to $p p \rightarrow W^{+} W^{-} \rightarrow 4$ leptons at the LHC, JHEP 06 (2016) 065 [hep-ph/1605.03419]

[9] G. D'Agostini, A multidimensional unfolding method based on Bayes' theorem, Nucl. Instr. Meth. A 362 (1995) 487

[10] G. D'Agostini, Improved iterative Bayesian unfolding, 2010, physics.data-an/1010.0632 